\title{
Topological domain structure of the Escherichia coli chromosome
}

\author{
Lisa Postow, ${ }^{1}$ Christine D. Hardy, ${ }^{1}$ Javier Arsuaga, ${ }^{1,2}$ and Nicholas R. Cozzarelli ${ }^{1,3}$ \\ ${ }^{1}$ Department of Molecular and Cell Biology and ${ }^{2}$ Department of Mathematics, University of California at Berkeley, \\ Berkeley, California 94720, USA
}

\begin{abstract}
The circular chromosome of Escherichia coli is organized into independently supercoiled loops, or topological domains. We investigated the organization and size of these domains in vivo and in vitro. Using the expression of $>300$ supercoiling-sensitive genes to gauge local chromosomal supercoiling, we quantitatively measured the spread of relaxation from double-strand breaks generated in vivo and thereby calculated the distance to the nearest domain boundary. In a complementary approach, we gently isolated chromosomes and examined the lengths of individual supercoiled loops by electron microscopy. The results from these two very different methods agree remarkably well. By comparing our results to Monte Carlo simulations of domain organization models, we conclude that domain barriers are not placed stably at fixed sites on the chromosome but instead are effectively randomly distributed. We find that domains are much smaller than previously reported, $\sim 10 \mathrm{~kb}$ on average. We discuss the implications of these findings and present models for how domain barriers may be generated and displaced during the cell cycle in a stochastic fashion.
\end{abstract}

[Keywords: Supercoiling; topological domains; microarrays; electron microscopy; bacterial chromosome]

Received March 29, 2004; revised version accepted May 13, 2004.

The Escherichia coli chromosome is $4.6 \mathrm{Mb}$ long, circular, and contains a single origin of replication. These characteristics present challenges for replication and segregation, which can nonetheless be completed as quickly as every $20 \mathrm{~min}$. In addition, the chromosome must be compacted $\sim 10^{3}$-fold to fit inside the cell (Holmes and Cozzarelli 2000), and its structure must ensure that entanglements between replicated sister chromosomes and subsequent guillotining at the septum are prevented. Thus, the bacterial chromosome is folded in a manner that confers a high level of both organization and compaction (Sherratt 2003).

A fundamental aspect of the folding and compaction of chromosomal DNA is its negative supercoiling (Holmes and Cozzarelli 2000), which is also critical for the numerous processes that require unwinding of DNA (Funnell et al. 1986; Pruss and Drlica 1989|. Even a modest reduction of negative supercoiling in bacteria is lethal (Zechiedrich et al. 1997). Supercoiling, however, is fragile. Without additional stabilization, just one DNA break would relax the entire chromosome and kill the cell. Replication itself causes relaxation, as only intact DNA can be supercoiled, and the newly synthesized DNA strands are complete only upon the conclusion of replication.

${ }^{3}$ Corresponding author.

E-MAIL ncozzare@socrates.berkeley.edu; FAX (510) 643-1079.

Article and publication are at http://www.genesdev.org/cgi/doi/10.1101/ gad.1207504.
Fortunately, the effects of DNA interruptions are limited by the binding of proteins, and perhaps RNAs, throughout the chromosome (Drlica 1987). These connections divide the chromosome into topologically isolated loops, that is, domains, which are the subject of this report. The operational definition of a domain is the region relaxed by an interruption in the DNA. We do not explicitly consider loops of chromosomal DNA that are not topologically constrained, but we present evidence below that bacterial chromosome loops are typically isolated topologically.

There is both in vitro and in vivo evidence for topological domains. Chromosomes isolated from E. coli cells were found early on to be folded by unconstrained negative supercoiling (Worcel and Burgi 1972). Unlike plasmids, which are relaxed by a single nick, a number of nicks were required to relax the chromosome completely. The conclusion from this early work was that chromosomes are divided into 12-80 domains. Similar results were found using a different method to measure chromosome relaxation in vivo (Sinden and Pettijohn 1981). Based on these experiments, domains were estimated to be $\sim 100 \mathrm{~kb}$ in length, requiring each genome to be divided into $\sim 50$ domains.

Morphological evidence for the existence of topological domains was provided by electron microscopy (Kavenoff and Bowen 1976; Kavenoff and Ryder 1976). Isolated E. coli chromosomes display many individually supercoiled loops emanating from a central region. These supercoiled loops were hypothesized to be topological do- 
mains. This view was supported by the finding that a single loop could be relaxed while the rest of the loops remained supercoiled (Delius and Worcel 1974). Moreover, Kavenoff and coworkers (Kavenoff and Bowen 1976; Kavenoff and Ryder 1976) counted the number of loops visible by electron microscopy and estimated between 65 and 200 loops per nucleoid, in rough agreement with the above estimates for the number of domains.

Although these studies were consistent with the existence of topologically closed domains, there were shortcomings to the methods that have bred some skepticism. For example, the in vitro domain barriers might have been created during chromosome isolation. A giant, highly compacted, twisted DNA could easily have acquired artifactual cross-links through bidentate binding by proteins or RNAs. Conversely, domain barriers that rely on transient attachments could be lost during chromosome isolation. The study of domains in vivo relied on measurements of relatively small differences in trimethylpsoralen binding, an unreliable technique (Sinden et al. 1980; Bliska and Cozzarelli 1987). In addition, the calculation of domain size in the studies cited above was based on the assumption that all domains are equal in size, but there is no evidence to support this view. In spite of these uncertainties, domains are presumed to exist, and the commonly cited figure for their size is 50-100 kb (Drlica 1987; Pettijohn 1996; Trun and Marko 1998).

More recent experiments have suggested that this much-cited domain size is too large. A less invasive approach to studying domains in vivo was taken by Higgins et al. (1996), who used the requirement for negative supercoiling of the $\gamma \delta$ resolvase DNA substrate to study domain structure in a region of the Salmonella enterica chromosome. They concluded that topological domains are variable in both size and the placement of barriers, and that domains average $25 \mathrm{~kb}$ in length. Although these studies had the advantage of relying neither on the isolation of intact chromosomes nor on the estimation of the efficiency of nicking the genome, there are caveats to this method that could lead to an overestimation of domain size, raising the possibility that domains are even smaller (see Discussion).

Four basic models for how domains might be organized in the bacterial cell are illustrated in Figure 1A. Combinations of these models are also possible. For simplicity, we have shown each chromosome as divided into only six domains. We have depicted three separate chromosomes for each model and have included a marker gene at a specific site, in red, to indicate the sequence specificity of domain barrier placement. These models differ in terms of two unanswered questions regarding domain organization. First, are domain barriers placed at specific sites in the genome? For models in which the placement of the barriers is fixed (models I and III), the distance of the marker gene to its nearest domain barrier is the same in all chromosomes. Conversely, when the site of barriers is variable (models II and IV), the distance of the marker gene to its nearest domain barrier will also vary. Second, are domains of uniform size? If domain
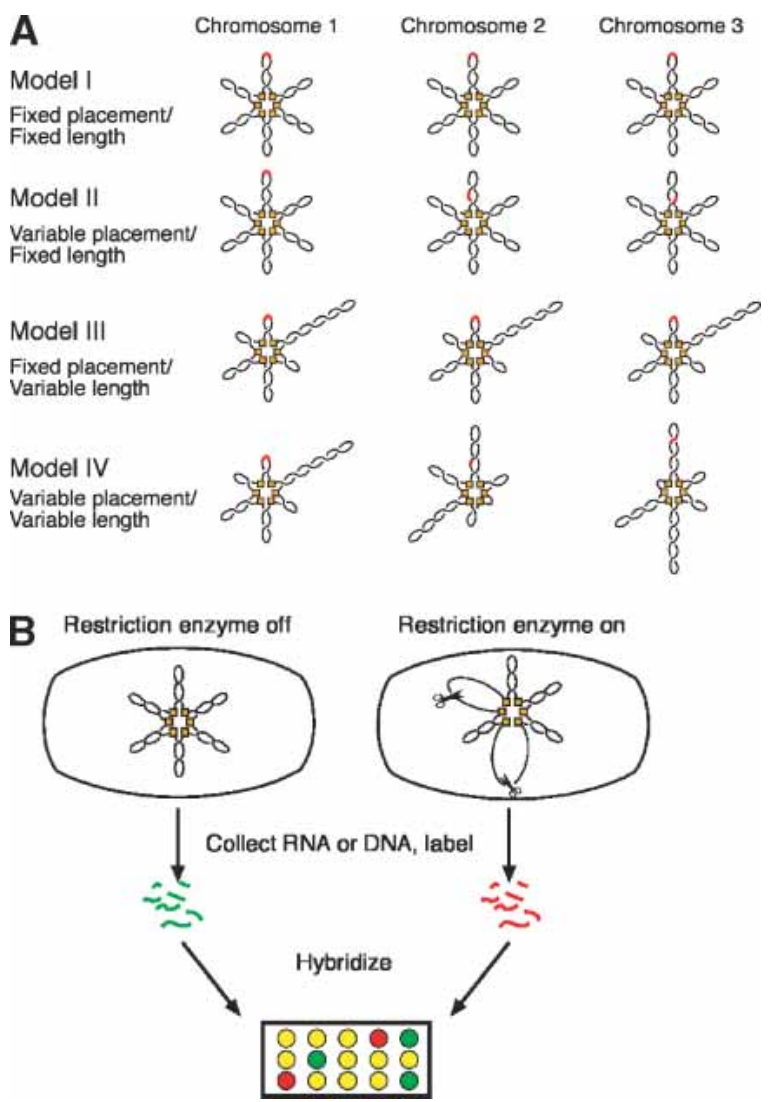

Figure 1. (A) Models for domain organization. Four models are presented that differ in the variability of barrier placement and domain length. For each model, three different chromosomes are shown, which may depict chromosomes in different cells or in the same cell at different times. Domain barriers are indicated with yellow boxes. A marker gene is shown in red to demonstrate the variability in barrier position. For simplicity, we have divided each chromosome into only six domains. $(B)$ Experimental scheme for microarray experiments. Cleavage of the chromosome will relax domains containing the cut site but leave uncut domains negatively supercoiled. DNA relaxation at numerous sites around the chromosome can be quantified using the expression of supercoiling sensitive genes (SSGs). To measure the expression of SSGs following cleavage, RNA was collected from cells prior to or following the expression of an active restriction endonuclease, labeled with either the dye Cy3 (green, reference) or Cy5 (red, sample), and used to probe microarrays containing PCR products from ORFs in the E. coli genome. The ratio of red to green signal measures the relative abundance of the transcripts from a particular gene. A yellow signal indicates about equal abundance of the reference and sample RNAs. To measure degradation from cut sites, genomic DNA was isolated from cells instead of RNA.

length is fixed (models I and II), the distance of the marker gene to its nearest barrier can never exceed half of that fixed length. However, if domain length is variable (models III and IV), the marker gene may be any distance from its nearest barrier.

We investigated the organization of topological domains and chromosome structure in $E$. coli both in vivo and in vitro. First, we showed that unlike in higher or- 
ganisms, the chromosome in vivo is generally accessible along its entire length to restriction enzymes, and thus there are no extensive stably bound structural components. Next, we used gene microarrays to examine the entire chromosome at once to determine how far DNA relaxation extends from double-strand breaks generated in vivo by a restriction enzyme (Fig. 1B). To measure relaxation, we monitored the transcription of $>300$ genes that respond to DNA relaxation (B.J. Peter, J. Arsuaga, A.M. Breier, A.B. Khodursky, P.O. Brown, N.R. Cozzarelli, in prep.). We then compared the results with Monte Carlo simulations of the four basic models in Figure 1A using varying mean domain lengths. Finally, we studied directly the sizes of supercoiled loops from isolated chromosomes using electron microscopy. Our results show that domains have a range of sizes, that domain barriers are not located sequence-specifically, and that chromosomes are structurally fluid, as in model IV (variable placement/variable length). In addition, we demonstrate that domains are much smaller than previously believed, with an average length of $\sim 10 \mathrm{~kb}$. We discuss the implications of these results for chromosome structure and function.

\section{Results}

The E. coli chromosome is generally accessible to restriction enzymes in vivo

We first gauged the accessibility of the E. coli chromosome to enzymatic cleavage in vivo. If chromosome structural elements protect a substantial fraction of the bacterial DNA from cleavage, much of the chromosome could not be assayed in our system. This is the case in eukaryotes: Nucleosomes and other binding proteins occlude eukaryotic chromosomal DNA from digestion (Clark and Felsenfeld 1971; Poirier and Marko 2002).

To study the vulnerability of the chromosome to restriction enzyme cleavage, we shifted cells containing a temperature-sensitive allele of the EcoRI nuclease to the permissive temperature, $32^{\circ} \mathrm{C}$ (Heitman et al. 1989). We chose EcoRI for this experiment because the high number of sites (645, spaced an average of $7 \mathrm{~kb}$ apart) would widely sample the genome. After $4 \mathrm{~h}$ at $32^{\circ} \mathrm{C}$, genomic DNA was isolated, labeled, and used to probe microarrays of the E. coli genome. For a reference, we used genomic DNA from cells harvested immediately before the shift to the permissive temperature. Because exonucleases process double-strand breaks generated by EcoRI in vivo (Heitman et al. 1989), genes near a double-strand break will be underrepresented, indicating the efficiency of breakage.

Four hours at the permissive temperature allowed time for significant exonuclease degradation. The $\log _{2}$ of the relative amount of each open reading frame (ORF) after cleavage was plotted versus the distance to its nearest EcoRI site (Fig. 2A). Genes within $3 \mathrm{~kb}$ of an EcoRI site have lower abundance due to DNA degradation. To analyze the fraction of individual EcoRI sites that was cleaved, we plotted the relative abundance of each ORF in sequential order along the chromosome and compared
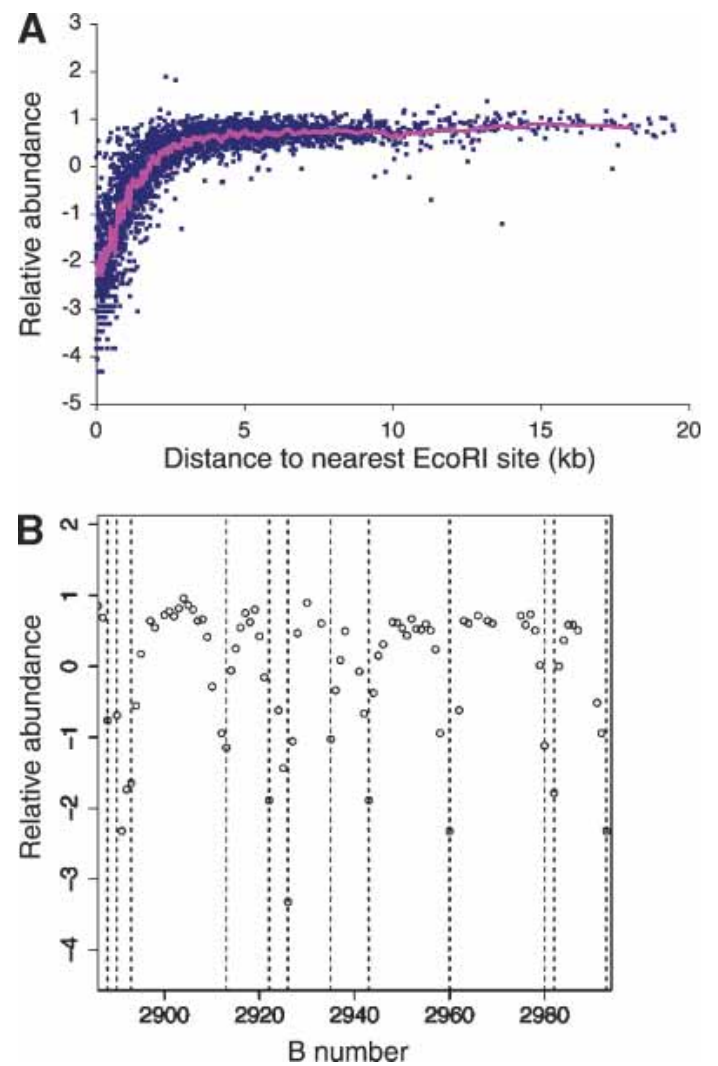

Figure 2. EcoRI cleavage in vivo. Shown are data taken $4 \mathrm{~h}$ after the shift to the permissive temperature for EcoRI. $(A)$ The $\log _{2}$ of the relative abundance of each ORF following the shift to the permissive temperature is plotted versus the distance of each ORF to its nearest EcoRI site (blue dots). A moving average over a window size of 30 data points is shown in purple. Window sizes in this and in subsequent figures have been optimized to yield a faithful display of the data (judged by comparison with smaller window sizes), while minimizing random fluctuations. The data were normalized such that the average ratio in the red and green channels over all ORFs is 1 . Because of DNA degradation, the ratio for intact ORFs is 1.6 , or 0.7 on the $\log _{2}$ scale shown. (B) DNA abundance data (circles) from a segment of the genome in $A$ replotted with respect to the $\mathrm{B}$ numbers of the genes that indicate their linear order along the chromosome (Blattner et al. 1997). Vertical dotted lines indicate the closest ORF to each EcoRI site. In the plot shown, the DNA from every gene that falls on a dotted line is either a local minimum or is present at less than half the level of the average of all genes. Therefore, all 12 genes in this plot show degradation from the cut site.

the relative abundance of ORFs that were very close to EcoRI sites with those farther away. A representative segment of the chromosome is shown in Figure 2B with the closest ORF to each EcoRI site indicated by a dotted vertical line. In this interval, all 12 genes that fall on dotted lines are local minima of DNA abundance and/or have a relative abundance less than half the average over the entire genome. In the entire genome, $86 \%$ of all genes closest to EcoRI sites (554 out of 645 sites) satisfied these criteria. We conclude that a large majority of sites are cleaved. 
This conclusion was confirmed by Southern blots of the DNA containing three specific EcoRI sites. The cleavage was complete, even after only a 30 -min EcoRI induction (data not shown), indicating that those sites were cut in all cells. Therefore, all or nearly all of the genomic DNA is accessible to restriction in every cell.

Because of the high number of EcoRI sites in the E. coli chromosome, few genes reside $>10 \mathrm{~kb}$ from a cut site. Our assay to study topological domains in vivo depends upon a comparison of the expression of genes in relaxed and supercoiled domains. Therefore, we used the lessfrequent cutter, SwaI, to assay a larger range of possible domain sizes.

SwaI efficiently cleaves the E. coli chromosome in vivo, but exonuclease degradation is not extensive

SwaI cleaves the E. coli genome much less frequently than EcoRI (117 SwaI sites versus 645 EcoRI sites) and therefore will relax some domains while leaving others supercoiled. SwaI was expressed in E. coli cells using the pLT7K vector (Kong et al. 2000), which minimizes leaky expression of lethal enzymes during the uninduced state (see Materials and Methods).

To determine the kinetics of SwaI cleavage in vivo, we treated isolated chromosomal DNA with PvuI and probed for the cleavage of a particular SwaI site that is
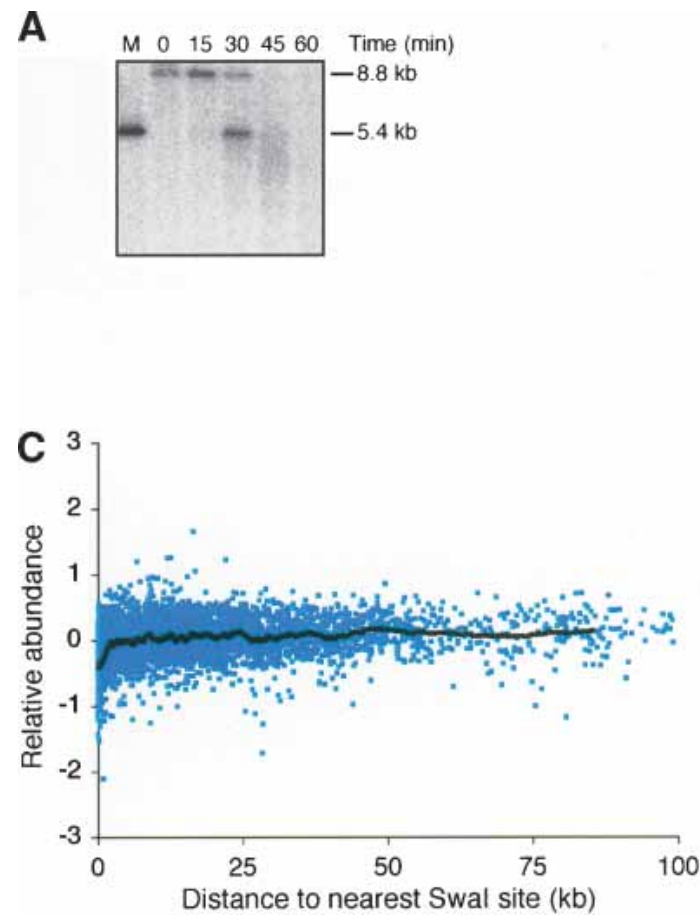

Figure 3. Cleavage of genomic DNA by SwaI in vivo. (A) SwaI was induced and genomic DNA was isolated at the times indicated, cleaved with PvuI, run on an agarose gel, and Southern blotted. The probed DNA is a PvuI fragment that contains a SwaI site. Digestion with SwaI cleaves the original 8.8-kb PvuI fragment into products with lengths of 3.4 and $5.4 \mathrm{~kb}$. The probe hybridizes only to the 5.4-kb fragment. M: Genomic DNA from the 0-min time point digested in vitro with both SwaI and PvuI. $(B)$ Quantification of the results from $A$. $(C, D)$ Genomic DNA was isolated from cells after SwaI induction and used to probe microarrays. $(C)$ The $\log _{2}$ of the relative abundance of each ORF after $45 \mathrm{~min}$ of SwaI induction is plotted relative to the distance of each ORF to its nearest SwaI site (blue dots). A moving average over 80 points is in black. $(D)$ The moving averages (over 80 points) are shown for the relative abundance of ORFs $30 \mathrm{~min}$ (turquoise), $45 \mathrm{~min}$ (black), and $60 \mathrm{~min}$ (purple) following SwaI induction.

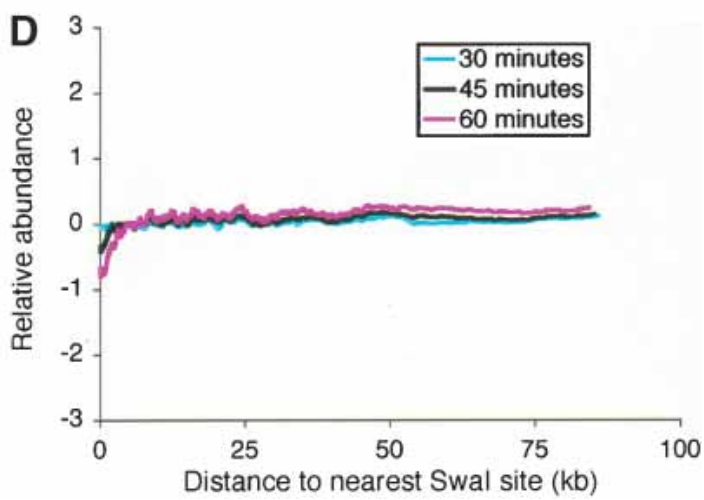

flanked by nearby PvuI sites (Fig. 3A). A 5.4-kb cleavage product appeared after $15 \mathrm{~min}$ of SwaI induction, and by (Fig. 3B). Thus, as with EcoRI, cleavage is very efficient. No cleavage was detected in an otherwise isogenic strain not express SwaI (data not shown). A smear o frer shorter than $5.4 \mathrm{~kb}$ was visible, particularly after 45 min of induction, indicative of DNA degradation by exonucleases in vivo (Fig. 3A). To gain a more globa of chromosome cleavage, we used pulsed field gel shown detected after $15 \mathrm{~min}$ of SwaI expression, and restriction was nearly complete by $45 \mathrm{~min}$.

We next assessed quantitatively the extent of exonuclease degradation following restriction cleavage by SwaI using microarrays. Degradation of the genes we to use as local probes of supercoiling would deconfession and confound the measure of doabundance of each ORF 45 min following SwaI induction is plotted against the distance to the nearest SwaI site, along with a moving average through the data. In Figure $3 \mathrm{D}$, the moving averages for the results 30,45 , and 60 min after induction are plotted to indicate the degradation over time. Exonuclease degradation is virtually undetectable 30 min following induction and extends only

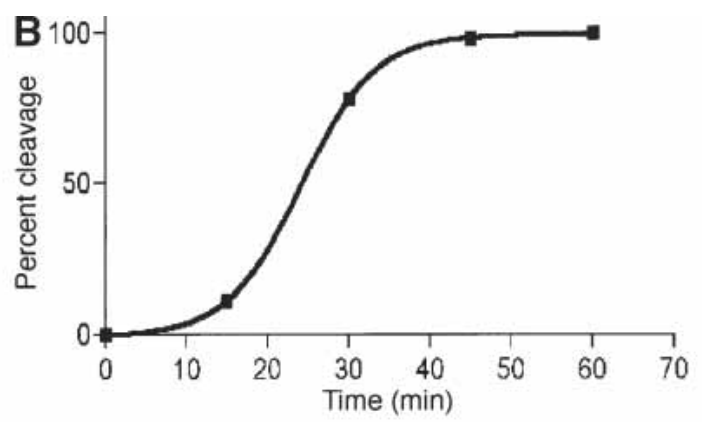
45 min, cleavage of the SwaI site was nearly complete 
Postow et al.

a few kilobases 60 min after induction; in all cases there is less than a twofold change in abundance. The pattern of degradation is similar for the supercoiling sensitive genes (data not shown). Because we do not allow digestion to proceed for more than an hour in our experiments, DNA degradation does not undermine measurement of topological domain size in vivo.

\section{Transcription of SSGs close to SwaI sites responds to DNA relaxation}

To study how far relaxation extends from a doublestrand break, we measured using microarrays the relative expression of the supercoiling sensitive genes (SSGs) following chromosome cleavage with SwaI (Fig. 1B). An SSG should respond to DNA cleavage only if it is in the same domain as the break. In the extreme case of no domain barriers, expression of all SSGs would be affected. RNA from preinduction cells was used as a reference. The $\log _{2}$ of the expression ratio for each gene is plotted relative to the distance to its nearest SwaI site in Figure 4 . Genes with a positive value are induced compared to the reference, whereas those with a negative value are repressed.
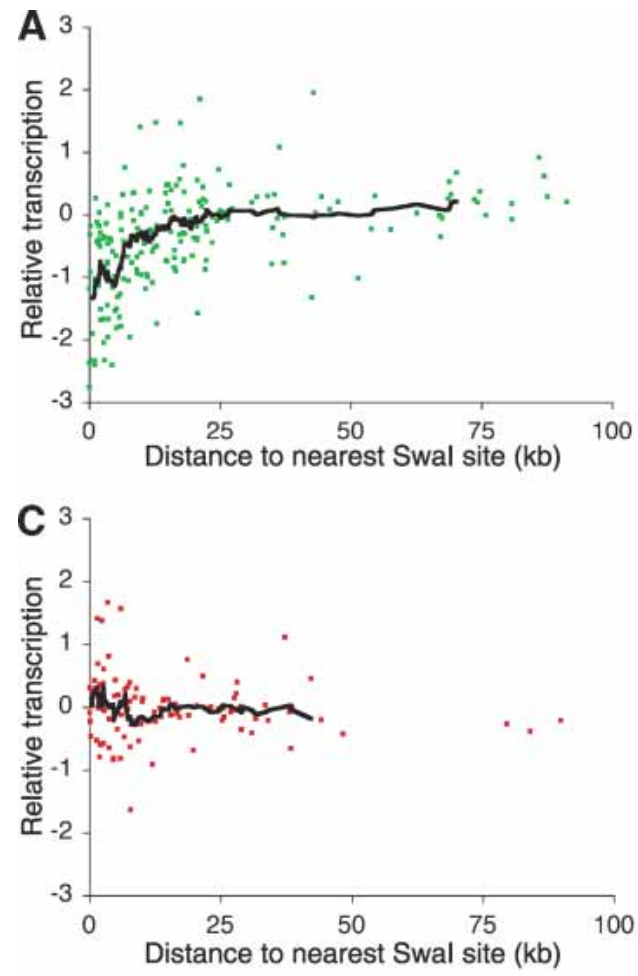

We first analyzed the relaxation-repressed genes, which are more abundant than the relaxation-induced genes (195 repressed vs. 106 induced). In Figure 4A, we show the raw data and moving average for the 45-min time point, when restriction is close to $100 \%$ but degradation is minimal (see Fig. 3). In Figure 4B, we show the moving averages alone 30, 45, and $60 \mathrm{~min}$ after SwaI induction. Forty-five min after induction, the genes close to a SwaI site are clearly repressed and the repression decreases to zero as the distance from a SwaI site increases. The mean repression level within $5 \mathrm{~kb}$ of a SwaI site is twofold, similar to that obtained upon gyrase inhibition (B.J. Peter, J. Arsuaga, A.M. Breier, A.B. Khodursky, P.O. Brown, and N.R. Cozzarelli, in prep.). The 30min time point showed less and the 60 -min time point slightly more repression than the 45 -min time point (Fig. $4 \mathrm{~B})$, but the overall pattern remained the same. Importantly, the repression spreads much farther from the SwaI sites than expected from DNA degradation alone (cf. Figs. 3C and 4A). Therefore, our results demonstrate that topological domain barriers indeed exist because DNA relaxation occurs near cut sites but not farther away. In the absence of such barriers, SSGs would be relaxed regardless of their proximity to a cut site.

We next investigated the expression of relaxation-in-
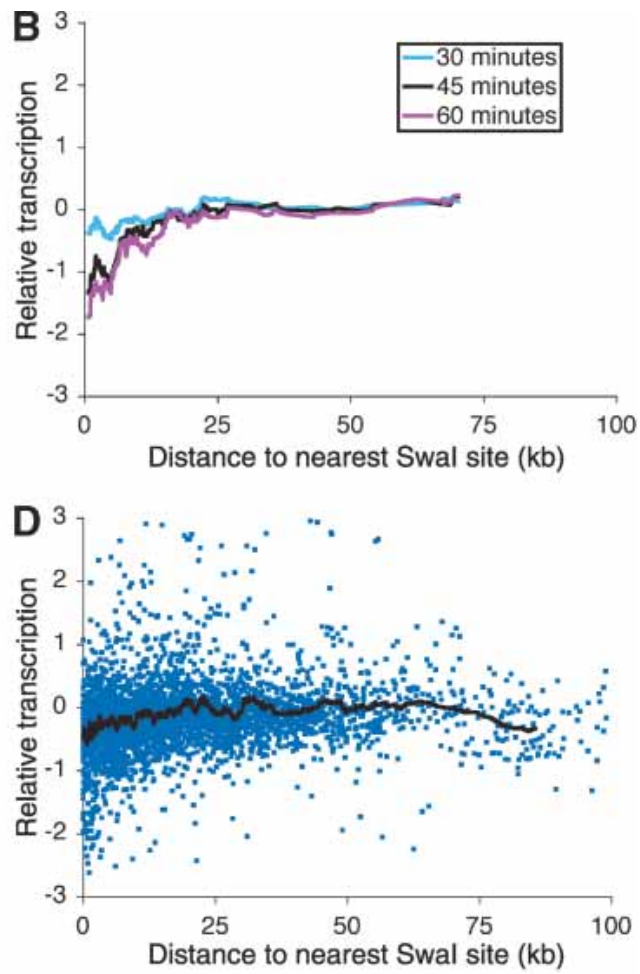

Figure 4. Gene expression in vivo following cleavage by SwaI. RNA was isolated from cells following SwaI induction and used to probe microarrays. The $\log _{2}$ of the relative transcription of genes was plotted relative to the distance of each gene to its nearest SwaI site. $(A, B)$ The expression of relaxation-repressed genes. $(A)$ The expression of each relaxation-repressed gene following $45 \mathrm{~min}$ of SwaI induction is indicated by green dots. A moving average over 20 points is shown in black. $(B)$ The moving averages (over 20 points) are shown for the 30-min (turquoise), 45-min (black), and 60-min (purple) time points. The expression of relaxation-induced genes following $45 \mathrm{~min}$ of SwaI induction is plotted with a 10-point moving average through the data in C, and the expression of all non-SSGs following 45 min of SwaI induction is plotted with an 80-point moving average in $D$. 
duced genes $45 \mathrm{~min}$ after induction of SwaI (Fig. 4C). Although the relative scatter in these data is greater than that for the relaxation-repressed set because of the smaller number of genes, transcription of the relaxationinduced genes is induced by SwaI expression. This trend is in contrast to the general repression of genes across the genome (Fig. 4D) and despite possible repressive effects of DNA degradation (Fig. 3C).

To evaluate the statistical significance of these data, we plotted the expression ratios of all genes that are not on the SSG list as a control (Fig. 4D). The moving average dips slightly close to SwaI sites, likely due to a combination of nuclease activity and low-level repression by relaxation of many genes that, nevertheless, do not fit the strict criteria of the SSG list. The repression and induction of SSGs relative to non-SSGs after cutting are highly statistically significant, despite the scatter in the data inherent to microarray-based gene expression analysis. The relative repression of relaxation-repressed genes within $10 \mathrm{~kb}$ of a cut site had a p-value of $<0.0001$, and the $\mathrm{p}$-value for induction of relaxation-induced genes was 0.0006 . We further analyze quantitatively the repression data below.

\section{Domains barriers are not fixed}

In the above experiments, we looked at many genes, each in its own unique SwaI environment, to study the topo- logical organization of the entire chromosome. However, this analysis does not reveal whether domain barriers are placed stably at the same sites in all cells. To answer this question and to provide a higher-resolution view of one region of the chromosome, we took advantage of the 204kb SwaI fragment between minutes 54 and 59 on the chromosome that contains several SSGs that are repressed by relaxation (Fig. 5A). We examined two SSGs in the center of this long region, $\operatorname{dap} A$ and $x \operatorname{se} A$, which are each $>80 \mathrm{~kb}$ from the nearest SwaI site and are not repressed upon SwaI induction. We then created strains with SwaI sites incorporated into five locations (labeled A-E in Fig. 5A) in varying proximity to these reporter genes, such that each strain had one ectopic SwaI site.

The effect of the ectopic SwaI sites on the expression of the reporter genes helps discriminate between fixed (models I and III) and variable (models II and IV) placements of domain barriers. If domain barriers were located at specific sequences in all cells, the reporter genes would be repressed to the same level by cutting at any site within a given domain, irrespective of how far the gene is from the cut site. Conversely, when the gene and the cut site are in different domains, expression of the gene would remain at the nonrepressed level regardless of the distance between the cleaved site and the reporter gene. Therefore, a sharp drop in expression of the reporter gene would mark the transition of an ectopic SwaI site from an adjacent domain to the same domain as the
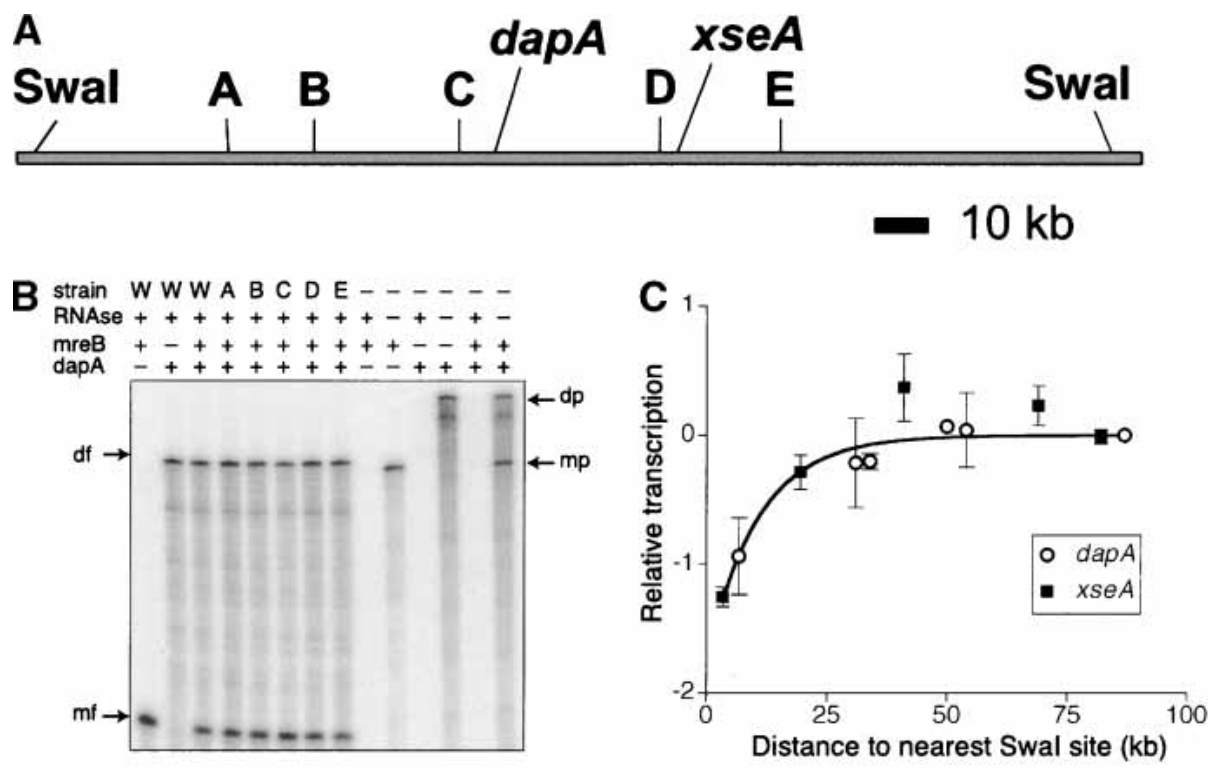

Figure 5. Effect of ectopic SwaI sites on the expression of two relaxation-repressed genes. $(A)$ Strains were constructed in which one of five SwaI sites (A-E) was inserted within a 204-kb region devoid of endogenous SwaI sites. The positions of the boundary SwaI sites, as well as the positions of the relaxation-repressed genes dapA and $x s e A$, are shown. A 10-kb size reference is indicated. (B) RNA from the wild-type strain $(\mathrm{W})$ and from strains containing one of the ectopic SwaI sites (A-E) was incubated in the presence $(+)$ or absence $(-)$ of ${ }^{32} \mathrm{P}$-labeled probes for dapA and $m r e B$, treated with RNase, and run on a denaturing polyacrylamide gel. Control lanes lacking RNase (-) were also included. Indicated are the positions of the dapA probe (dp), the mreB probe (mp), the dapA protected fragment (df), and the $m r e B$ protected fragment (mf). Measurements of $d a p A$ and $x s e A$ transcription were performed in duplicate, and the results, quantified using a PhosphorImager, are shown in $C$. All values are normalized to the internal mre $B$ control, and expressions are relative to that in the wild-type strain for a given experiment. An exponential curve is shown, indicating an average domain size of $11 \mathrm{~kb}$. Error bars indicate one standard deviation. The order of values along the $X$-axis, from left to right, correspond to strains $\mathrm{D}, \mathrm{C}, \mathrm{E}, \mathrm{D}, \mathrm{B}, \mathrm{C}, \mathrm{A}$, $\mathrm{E}, \mathrm{B}, \mathrm{W} / \mathrm{A}$, and $\mathrm{W}$. 
SSG. If, however, domain barriers have variable placements, we expect a gradual decrease in expression of the reporter gene as SwaI sites are moved closer, because there is always some probability of an intervening domain barrier.

RNA was isolated from cells following $45 \mathrm{~min}$ of SwaI induction in the wild-type strain as well as in the five strains with ectopic SwaI sites. We used RNase protection to quantify the expression of $\operatorname{dap} A$ and $x s e A$. For an internal control, we used the transcription of mreB, which is located far away from the region under study and thus should be unaffected by the inserted SwaI sites. Moreover, expression of $m r e B$ is not altered by DNA relaxation. An experiment using the $\operatorname{dap} A$ probe is shown in Figure 5B.

After normalization to $m r e B$, we compared SSG expression in the wild-type strain to that with inserted SwaI sites. As predicted by variable barrier placement models, there was a gradual decrease in repression as the site was moved farther from the gene (Fig. $5 \mathrm{C}$ ). This pattern was observed for both the $\operatorname{dap} A$ and the $x \operatorname{se} A$ genes. The data fit well an exponential function, as required by model IV, and yielded an average domain size of $11 \mathrm{~kb}$. We conclude that domain barriers are not placed at the same sites in all cells.

\section{Expression of relaxation-repressed genes after SwaI cutting reflects small domains of variable size}

Because domain barriers are not placed at fixed sites, we do not consider further models I and III (Fig. 1A). We next sought to determine whether domain sizes are fixed or variable to discriminate between models II (fixed domain length) and IV (variable domain length). To this end, we analyzed quantitatively the pattern of SSG expression changes with respect to distance from a cut site and compared it to Monte Carlo simulations of the two contending models. We used only the larger relaxation-repressed microarray data set for this analysis. We varied in the simulations the average domain size between 6 and 46 $\mathrm{kb}$ to obtain the best fit to the data.

To ease comparisons between simulated and experimental data, we binned the two data sets in the same way and averaged the binned values. We then normalized the expression results between 0 (supercoiled) and 1 (relaxed) and plotted them on a logarithmic scale as a function of distance to the nearest SwaI site. We generated a set of curves for each model by varying the average distance between domain barriers. The best fit to the binned microarray data for each model is shown in Figure 6A. The curve for model II drops off linearly, because the probability that a cut site and an SSG are in the same domain will decrease with the distance between them until it equals the size of a domain. Then, the probability of relaxation is zero. This behavior results in a curved pattern on the semi-log plot in Figure 6A. Model IV, which postulates domains of variable lengths, yields a first-order exponential decay curve and a straight line on a semi-log plot. The binned microarray fit well a straight line $\left(\mathrm{R}^{2}=0.98\right)$, in agreement with the model IV simula-
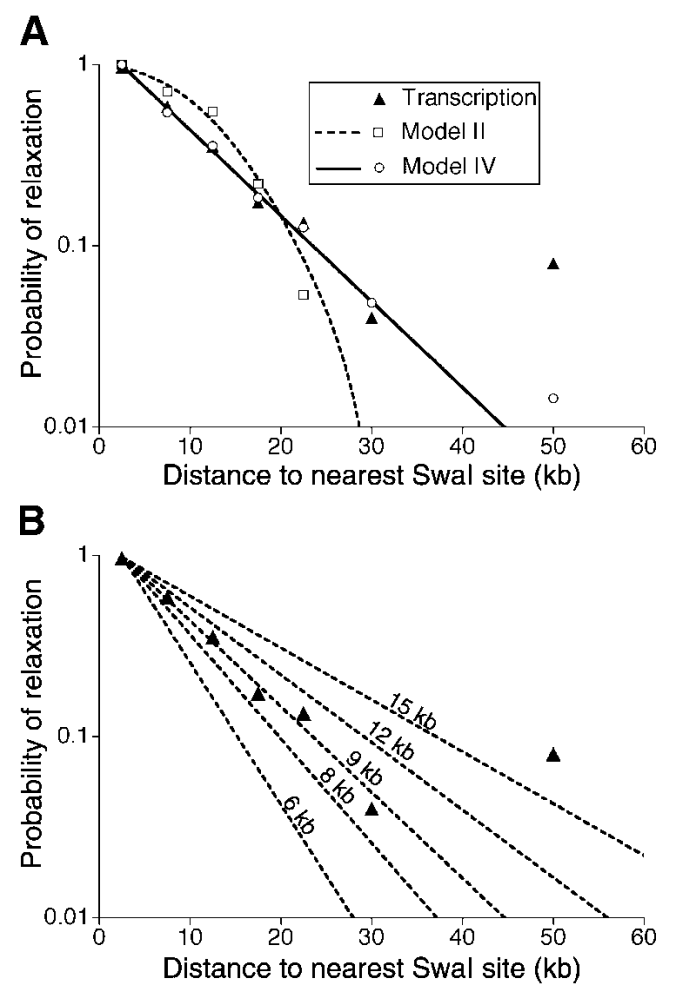

Figure 6. Comparison of transcription measured with microarrays to Monte Carlo simulations. The probability of an SSG being relaxed is plotted as a function of distance from a SwaI site. Measurements of the transcription of relaxation-repressed genes by microarrays $45 \mathrm{~min}$ following SwaI induction were binned in the following increments: $0-5 \mathrm{~kb}, 5-10 \mathrm{~kb}, 10-15 \mathrm{~kb}$, $15-20 \mathrm{~kb}, 20-25 \mathrm{~kb}, 25-35 \mathrm{~kb}$, and $35-65 \mathrm{~kb}$, and normalized such that most values are between 0 (unchanged) and 1 (repressed) so that the experimental and simulated data sets could be directly compared. These normalized values are equivalent to the probability of relaxation, and we have used this terminology for clarity and for comparisons in Figure 7. The average value for each bin is plotted at the midpoint of the binned distances. Experimental values for distances $>65 \mathrm{~kb}$ average to a negative value, and thus are excluded from these graphs. Values from Monte Carlo simulations for models II and IV were treated identically and plotted. $(A)$ The binned transcriptional microarray data (black triangles) and the best-fit values for model II (open squares) and model IV (open circles) simulation data are plotted on a semi-log graph. Both the model IV data and the transcription data fall on a straight line corresponding to an average domain size of $9 \mathrm{~kb}$. In contrast, the best-fit model II values correspond to a domain size of $23 \mathrm{~kb}$ and do not fit a straight line on this semi-log graph. The model II simulation values go to 0 when the domain length is exceeded, and this cannot be represented on the log scale shown. $(B)$ The transcriptional microarray data (black triangles) were compared to model IV simulations using average domain sizes that varied from $6 \mathrm{~kb}$ to $15 \mathrm{~kb}$, as indicated above each line. The data points fall between the best-fit lines for average domain sizes of 8 and 12 $\mathrm{kb}$, with the exception of the outlier in the last bin.

tion. The data can also be fit to the model II simulation predictions, albeit less well, but require a domain size of $23 \mathrm{~kb}$. This value is inconsistent with that obtained from the microscopy data presented below. As expected, the 
data do not fit well simulations of models I and III (data not shown). We conclude that the microarray data are most consistent with model IV.

We next used the model IV simulations to determine the average domain size by comparing the microarray data to simulations over a range of average domain lengths (Fig. 6B). The binned data fit best an average domain size of $9 \mathrm{~kb}$. We also fit an exponential decay curve to the raw scattered data shown in Figure 4A. The bestfit curve again indicates an average domain size of $9 \mathrm{~kb}$, with a $95 \%$ confidence interval between 6 and $16 \mathrm{~kb}$.

Sizes of supercoiled loops of chromosomes isolated in vitro are variable and small

As an independent measure of domain organization, we used electron microscopy of isolated chromosomes. Previous studies have shown that these resembled rosettes of supercoiled loops of DNA emerging from a central region, but these loops were much larger than the domain size that we measured (Delius and Worcel 1974;
Kavenoff and Bowen 1976; Kavenoff and Ryder 1976). To minimize preparation artifacts, we visualized the bacterial chromosome in as close to a native state as possible. We spread chromosomes directly from the peak fraction of a sucrose gradient onto glow-discharged carbon-coated grids for electron microscopy without adding any DNAbinding proteins or cross-linking agents. The high concentration of salt $(1 \mathrm{M} \mathrm{NaCl})$ and sucrose $(20 \%)$ was sufficient to keep the nucleoids intact for the short time before deposition onto the grid, as indicated by the absence of partial or broken chromosomes.

The chromosomes analyzed in this manner indeed showed rosettes of tightly supercoiled loops of DNA (Fig. 7A). The loops are evidently supercoiled because they are branched and highly kinked, as are supercoiled plasmids spread under identical conditions (Fig. 7B). Of the five chromosomes that were analyzed carefully, none showed relaxed loops or broken DNA, indicating that the procedure preserved the DNA intact. Individual loops in a single chromosome could be relaxed by $\gamma$ irradiation, whereas the remainder of loops remain supercoiled, demonstrating that topological substructure is
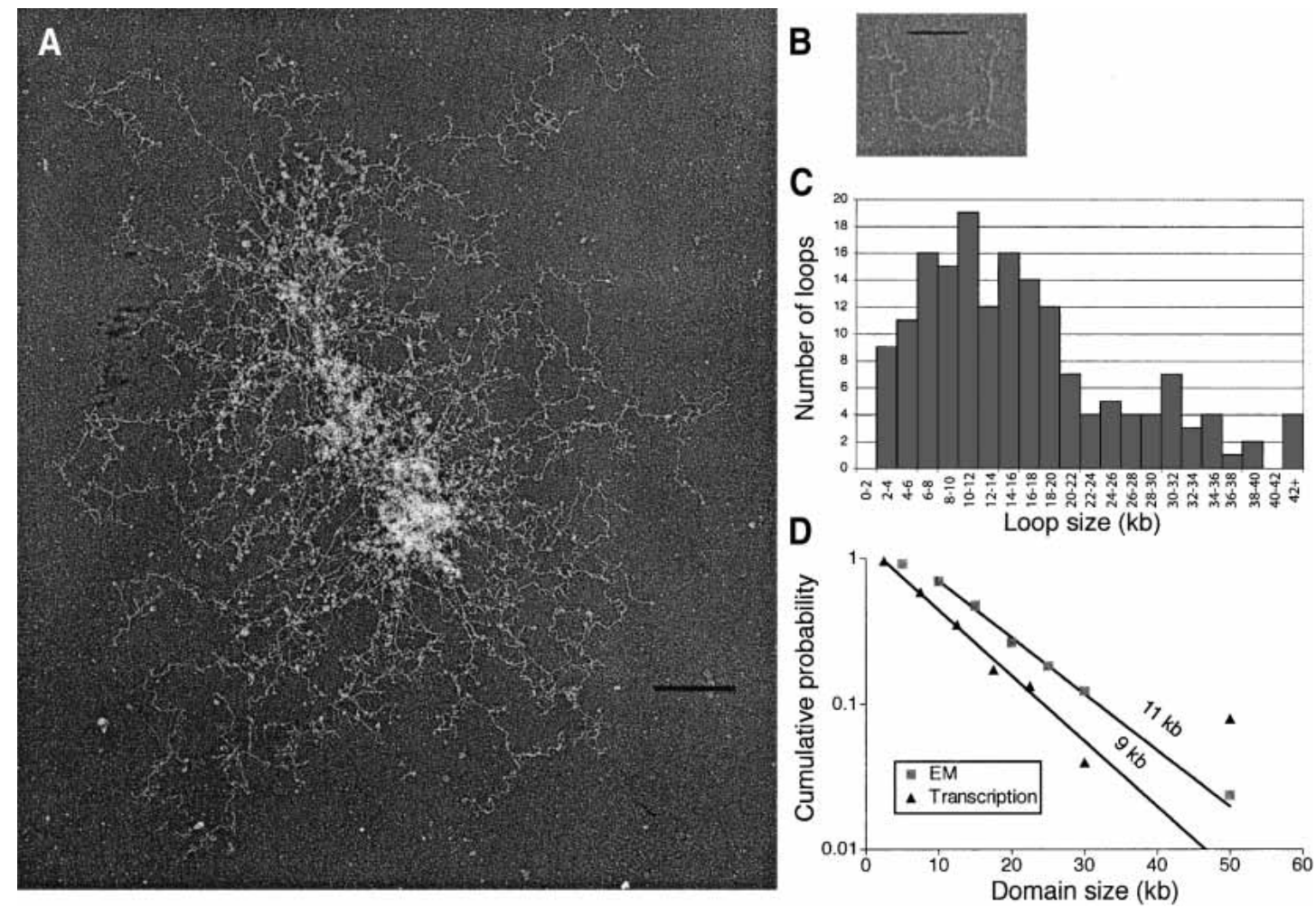

Figure 7. Supercoiled loop measurements from electron micrographs of isolated chromosomes. (A) E. coli chromosomes were gently isolated by sucrose gradient sedimentation and spread onto carbon-coated electron microscopy grids. A representative chromosome is shown, photographed at 12,000× magnification. Bar, $500 \mathrm{~nm}$. (B) A similarly treated 7-kb negatively supercoiled plasmid photographed at 50,000x magnification. Bar, $100 \mathrm{~nm}$. (C) Quantification of loop sizes from electron micrographs. We traced 169 clearly defined supercoiled loops and compared their lengths to that of a 7-kb plasmid. Loop sizes were binned in 2-kb increments and plotted as a histogram. (D) Comparison of domain sizes from microarray and electron microscopy (EM) experiments. The cumulative probability of loop sizes measured by EM (gray squares) and of domain sizes from transcriptional microarray experiments (black triangles) are plotted. Microarray data were binned as in Figure 6. The lines show the best-fit exponential function to each data set. Because loops $<10 \mathrm{~kb}$ were difficult to score by EM, the data points corresponding to these loop sizes were not included in fitting the exponential function. The best-fit exponential functions indicate a domain size of $9 \mathrm{~kb}$ for the microarray data and $11 \mathrm{~kb}$ for the microscopy data. The vertical displacement between the two data sets is due to the paucity of small loop measurements in the microscopy data set. 
maintained in our preparations (data not shown). Due to the gentle nature of our spreading technique, only $12 \%$ of the DNA on average was released from the central electron-dense mass. This mass was not seen in previous electron micrographs of chromosomes, perhaps because of the more extensive stripping of nucleoid components in these preparations and the addition of exogenous DNA-binding proteins that aid in spreading of the DNA onto the grid (Kavenoff and Bowen 1976; Kavenoff and Ryder 1976).

We measured the lengths of all clearly defined supercoiled loops from five different chromosomes, a total of 169 loops. As a reference, we measured the length of a supercoiled plasmid of known size that had been treated identically (Fig. 7B). The range of loop sizes was striking, spanning from 2 to $66 \mathrm{~kb}$ (Fig. 7C). This is a direct demonstration of the variable domain length of model IV. To analyze the distribution quantitatively, we excluded loops $10 \mathrm{~kb}$ and smaller, which would tend to blend into the central mass and therefore be underrepresented. We plotted the cumulative probability of loop length on a log scale (Fig. 7D). An exponential function fit very well to the data $\left(\mathrm{R}^{2}>0.99\right)$, as required by model IV. The average domain length calculated by this analysis is $11 \mathrm{~kb}$, with a $95 \%$ confidence interval between 10 and $13 \mathrm{~kb}$. This value is the same, within error, as that obtained with the transcriptional microarray data plotted in the same manner (Fig. 7D). The under-sampling of small loop sizes by microscopy causes a twofold upward displacement of the microscopy data from the microarray results.

The very good agreement of the functional data in vivo with the morphological results obtained in vitro provides strong evidence that model IV (variable placement/ variable length) describes domain organization in E. coli, and that the average domain length is $\sim 10 \mathrm{~kb}$.

\section{Discussion}

Supercoiling sensitive genes as in vivo probes of chromosomal structure

We developed a new method to study topological domains throughout the entire chromosome. We gauged supercoiling levels simultaneously at a large number of loci, the SSGs. These 301 genes were previously found by our laboratory to be either induced or repressed when the E. coli chromosome is relaxed by inhibition of DNA gyrase (B.J. Peter, J. Arsuaga, A.M. Breier, A.B. Khodursky, P.O. Brown, and N.R. Cozzarelli, in prep.).

The site specificity of restriction enzymes allowed us to test directly the sensitivity of SSGs to local topology. This analysis was necessary because inhibition of DNA gyrase has profound consequences in addition to DNA relaxation, including the cessation of DNA synthesis, leaving open the possibility that a significant fraction of the genes were not responding to DNA topology in the initial screen. However, we found that the expression of SSGs was in fact dictated by their proximity to a doublestrand break. The probability that the transcription of relaxation-repressed genes would diminish and that of relaxation-induced genes would increase within $10 \mathrm{~kb}$ of a double-strand break was highly significant ( $p \leq 0.0006$ ) compared to the set of non-SSGs. The effect on expression is not due to DNA degradation from the cleaved sites because the extent of DNA degradation is far less than the extent of transcriptional repression. Moreover, degradation is not appreciable until $60 \mathrm{~min}$ following SwaI induction, whereas transcriptional repression is clearly apparent $30 \mathrm{~min}$ earlier. Therefore, the SSGs are valid indicators of local topology and are effective tools for analyzing chromosomal topology in vivo.

\section{The bacterial chromosome is a fluid assortment of small, supercoiled loops}

Our studies, which involve in vivo and in vitro experiments as well as Monte Carlo simulations, led us to three major conclusions regarding domain organization in E. coli. First, domain barriers are not placed at the same sites in all cells; second, domains average $10 \mathrm{~kb}$ in length; and third, domain lengths vary in an exponential fashion. These conclusions indicate that model IV /variable placement/variable length) best describes topological domains in E. coli, and that domains are smaller than had been previously proposed.

The strongest evidence for the variability of domain barrier location was provided by the analysis of the expression of two specific reporter genes with variable distances to the nearest SwaI site. The probability of relaxation dropped off gradually with the distance to a doublestrand break, as expected if barriers are not fixed in position. Further support, albeit indirect, for the absence of a sequence-specific chromosome organization center was provided by the general accessibility of the genome to cleavage by a more frequent cutter, EcoRI, and to exonucleases.

Microarray, RNase protection, and electron microscopy experiments indicated that domains average between 9 and $11 \mathrm{~kb}$ in length. The excellent agreement among these very different assays strongly supports our conclusion that domains are significantly smaller than previously estimated. Note that because domain sizes vary randomly, their half-length is the mean domain size multiplied by $\ln (2)$. Thus, an average domain size of 10 $\mathrm{kb}$ requires that half of all domains are smaller than $7 \mathrm{~kb}$.

Our third conclusion, that domain sizes are randomly distributed, was strongly suggested by the microarray experiments and confirmed by the electron microscopy results. We concluded this because the probability of relaxing a gene decayed exponentially with its distance from a double-strand break, as did the cumulative probability of loop sizes determined directly by electron microscopy.

\section{Comparison with previous studies}

Previous studies of topological domains in E. coli concluded that domains were larger than we have found. Our in vitro and in vivo experiments indicate that domains average $10 \mathrm{~kb}$. We believe that the discrepancy 
between our results and earlier studies is due to improvements in methodologies.

The two chief previous means of studying topological domains in the living bacterial cell, trimethylpsoralen incorporation and $\gamma \delta$ resolvase activity, both have limitations (Sinden and Pettijohn 1981; Higgins et al. 1996). From the reduction in the amount of psoralen binding with $\gamma$ radiation-induced breaks, one can obtain only an average view of domain structure. Furthermore, the nicks induced by $\gamma$ rays are in unknown locations, thus precluding determination of variability in domain barrier placement among cells. Finally, this method has proved inaccurate in previous studies, leading to a large overestimate of the amount of free negative supercoiling in bacterial cells (Sinden et al. 1980; Bliska and Cozzarelli 1987). The $\gamma \delta$ resolvase method has the advantage of measuring domain barriers in specific regions of the chromosome and being less invasive. However, a positive signal is genomic DNA loss, so only nonessential regions can be assayed. Most of the chromosome, therefore, is closed to scrutiny by this method and only $2 \%$ of the genome has been studied. Moreover, resolution at any time over the several hours of the experiment will be scored as an absence of a domain barrier, and thus this method will overestimate the size of dynamic domains.

In our electron microscopy studies, we were careful to minimize disruptions to chromosome structure. We deposited isolated chromosomes directly from a sucrose gradient onto carbon-coated grids. This procedure was both fast and gentle. Unlike past studies, we did not wash our grids in ethanol, which may alter factors critical to domain binding, nor did we add cross-linking or DNA-binding agents. Finally, we measured the lengths of undisturbed supercoiled loops rather than relaxed loops (Kavenoff and Bowen 1976; Kavenoff and Ryder 1976). Significantly, we saw no relaxed loops out of the 169 carefully examined; all are topologically constrained. There was an approximately twofold variation in the number of domains spread per nucleoid using our method, but the average domain size was similar for all nucleoids. We conclude that the loops we measured are representative of the chromosome as a whole.

The resolvase results, like ours, support an essentially random distribution of domain barriers but indicated that domains are on average two to three times larger than our estimate. The dynamic nature of domain barriers may explain the discrepancy; the $25 \mathrm{~kb}$ domain size reported by Higgins et al. (1996) may reflect the frequency of more stable domain barriers. A less likely alternative is that repair of double-strand breaks introduces some domain barriers in our studies.

Although we have focused on the nature of topological domains in E. coli, it is important to note that domains are an organizing principle of chromosome structure throughout nature. It is the presence of domains in eukaryotes that causes linear chromosomes to have topological properties such as supercoiling and catenation (or braiding), which require the actions of topoisomerases to resolve (Champoux 2001). Intriguingly, results from pulling metaphase newt chromosomes in the presence of restriction enzymes indicate DNA cross-links $\sim 15 \mathrm{~kb}$ apart on average (Poirier and Marko 2002). Perhaps topological domains have a similar size in many organisms.

\section{Models for domain barrier formation and dispersal}

We propose that the E. coli chromosome is organized into topological domains by transiently associated factors. A domain barrier consists of an association between a site on the chromosome and a stationary cellular component such as the inner membrane or another site on the chromosome such that the intervening DNA is isolated topologically. This model does not require that any particular protein or RNA function primarily as a domain barrier. Fortuitous cross-linking could result from a variety of cellular activities such as transcription, translation, or replication, and such effects could explain the random placement of domain barriers. Indeed, nascent transcription and translation of membrane-binding proteins have been shown to form topological domain barriers (Liu and Wang 1987; Lynch and Wang 1993). Transcription from a highly active promoter, even in the absence of membrane translocation, can form a spatially fixed domain barrier (Scheirer and Higgins 2001; Deng et al. 2004), although it seems that relatively few promoters are sufficiently active to have this effect (Manna and Higgins 1999). Whereas barriers induced by transcription, translation, or replication will be fixed spatially at a multitude of sites, they are likely to be dynamic temporally throughout the cell cycle. Over a population of cells, dynamic barriers formed by cell cycle-dependent transcription or replication would appear random in our assays, even though they have some sequence specificity.

Finally, any tight DNA-binding protein that by itself or with another protein binds to more than one DNA site imposes topological domains on the chromosome. Topoisomerases, by binding unproductively at DNA crossings (Zechiedrich and Osheroff 1990), close off topological domains, as does the chromosome translocase FtsK (Aussel et al. 2002; P. Pease and N.R. Cozzarelli, unpubl.). The estimated 500 barriers per genome could form as the result of a combination of the above factors. This would be consistent with the observation that domains are larger in stationary phase, when the cell is more quiescent (Higgins et al. 1996). Separate populations of more and less stable domain barriers would also explain why some barriers remain intact during more disruptive chromosome preparations, whereas others do not (Kavenoff and Bowen 1976; Kavenoff and Ryder 1976).

However, chromosome organization is likely not left entirely to chance encounters. One attractive possibility is that barrier formation is keyed to replication. The region in front of the replication fork must be positively supercoiled and the region directly behind the replication fork is relaxed, while the rest of the chromosome is negatively supercoiled. Barriers must continually be removed ahead of the fork, and inserted behind, both for replication to continue and to allow the replicated DNA 
to become negatively supercoiled. During this process, new barriers behind the fork may be placed in different locations than before. DNA condensation and extension follows a similar dynamic. The proteins that condense DNA must be removed to make way for the replication proteins. After the passage of the replication fork, recondensation must occur quickly, as only a small region of the DNA can be decondensed at any one time (Holmes and Cozzarelli 2000). Since DNA condensation by proteins requires tight, bidentate binding, as does domain barrier formation, the two processes could be carried out by the same proteins. That is, condensins may also act as domainins. Indeed, MukBEF, the chief E. coli condensin, does close off topological domains (Case et al. 2004). In addition, both MukBEF and its Bacillus subtilis counterpart, SMC, are likely involved in the organization and compaction of the chromosome in vivo (Hiraga et al. 1989; Niki et al. 1991; Sawitzke and Austin 2000; Lindow et al. 2002). This model provides an economical way to coordinately control replication, barrier formation, and DNA condensation.

\section{The benefits of small, fluid topological domains}

There are significant advantages to small, dynamic topological domains in a bacterial chromosome. First, a small domain size reduces the amount of DNA that is relaxed by DNA breakage. This is important for bacteria, which require negative supercoiling for viability (Gellert et al. 1976, 1977). Relaxation results whenever a DNA strand is incomplete, a situation that arises not just when damage occurs, but also during DNA replication. Even if the replisome itself forms a domain barrier, the gaps in the lagging strand will lead to DNA relaxation. Replication occurs continuously in exponentially growing cells, but in a $10-\mathrm{kb}$ domain, a gene will be relaxed by replication for only $\sim 10 \mathrm{sec}$. Second, small domains will greatly simplify the problems of catenane and precatenane resolution following replication (Ullsperger et al. 1995; Schvartzman and Stasiak 2004). Small domains concentrate catenane links, making it easier for the decatenase, topoisomerase IV, to find the links between enormous chromosomes (Espeli et al. 2003). Concentration of catenane links will also increase the free energy of catenanes and, therefore, will drive decatenation toward completion (Vologodskii and Cozzarelli 1993). Third, organization of the chromosome into 500 small loops will greatly aid in the repair of double-strand breaks by keeping the two ends to be joined in proximity to each other. Proper localization of ends would be particularly important when multiple double-strand breaks are repaired by nonhomologous end-joining. Finally, small domains will contribute substantially to the compaction of the chromosome. Assuming a random coil model for DNA, the division of an unreplicated chromosome into $\sim 500$ loops would decrease its radius of gyration from $\sim 10 \mu \mathrm{m}$ to $<0.5 \mu \mathrm{m}$. This degree of compaction alone is sufficient to fit the chromosome into the bacterial cell (Trun and Marko 1998).

Because all the various activities of the prokaryotic cell cycle occur concomitantly, the problems inherent to the organization and compaction of prokaryotic DNA have long been confounding. The bacterial chromosome must be free enough for transcription and replication to occur continuously. However, at the same time the chromosome must be ordered and compacted to fit inside the cell, as well as to permit efficient decatenation, segregation, and repair. Small, loosely defined, reversible topological domains help to solve this paradox. They provide structure to the chromosome without imposing rigidity.

\section{Materials and methods}

\section{Plasmids and strains}

Plasmid pJH10(TS6), a gift from Joseph Heitman (Duke University Medical Center, Durham, NC), was used to express EcoRI selectively. It contains a mutant EcoRI gene that encodes a temperature-sensitive enzyme under its native constitutive promoter (Heitman et al. 1989). The plasmid was transferred into MG1655 cells to form strain LP014.

Plasmid pLT7K-SwaI (Kong et al. 2000) was a gift from Huimin Kong (New England Biolabs, Beverly, MA). It is a derivative of pLT7K in which the SwaI gene is between two convergent promoters to ensure high levels upon induction with minimal promoter leakage when not induced. The sense promoter is inducible by IPTG, whereas the antisense promoter $\left(\lambda P_{R}\right)$ is repressed by a temperature-sensitive allele of the $\lambda$ cI protein.

Strain LP140 is an MG1655 derivative in which the $\lambda$ DE3 prophage containing the gene for bacteriophage T7 RNA polymerase has been integrated into the E. coli genome (Novagen). LP140 contains two plasmids: pLT7K-SwaI and pLysP, a pACYC184 derivative containing a mutant T7 lysozyme gene, which increases the expression of SwaI (Kong et al. 2000).

Ectopically positioned SwaI sites were generated with the $\lambda$ RED system for site-specific chromosomal insertions (Yu et al. 2000). PCR primer pairs containing $50 \mathrm{nt}$ from the $E$. coli genome and $20 \mathrm{nt}$ from the kanamycin resistance gene were used to amplify the kanamycin resistance gene flanked by genomic sequence, such that homologous recombination inserts the gene at a specific location. Insertions were made between convergently oriented genes to minimize transcriptional effects of the insertion, and no genomic DNA was deleted. One primer in each pair contained a SwaI site between the genomic sequence and the kanamycin resistance gene. Cells expressing the $\lambda$ recombination proteins were transformed with these PCR products, and recombinants were selected on Luria Broth (LB) plates containing kanamycin. The kanamycin resistance gene and its linked SwaI site were transferred into the LP140 background by P1 transduction.

\section{Induction of restriction enzymes}

EcoRI cleavage was induced in strain LP014. Cells were grown in $\mathrm{LB}$ at $42^{\circ} \mathrm{C}$ to an $\mathrm{OD}_{600}$ of $\sim 0.7$, and then transferred to $32^{\circ} \mathrm{C}$ to allow for EcoRI expression. For SwaI induction, cultures of $\mathrm{LP} 140$ were grown in $\mathrm{LB}$ at $37^{\circ} \mathrm{C}$ to an $\mathrm{OD}_{600}$ of 0.1 . They were shifted to $33^{\circ} \mathrm{C}$, the permissive temperature for the $\lambda$ cI protein. After $30 \mathrm{~min}$, restriction was induced by addition of $4 \mathrm{mM}$ IPTG.

After induction, samples of cultures were quenched at $0^{\circ} \mathrm{C}$ with a one-tenth volume of phenol/ethanol $(1 / 20)$ to stop mRNA degradation (Bernstein et al. 2002). Cells were harvested by centrifugation, frozen in liquid nitrogen, and stored at $-80^{\circ} \mathrm{C}$. 


\section{RNA and DNA isolation and microarray hybridization}

RNA for both microarrays and RNase protection assays was isolated using the "hot phenol" method (Khodursky et al. 2003) and then treated with DNase I. For microarray experiments, RNA isolated from cells before IPTG induction was used as the reference. cDNA was made using the Superscript II reverse transcriptase (Invitrogen). Each reaction contained $16 \mu \mathrm{g}$ of purified RNA with $10 \mu \mathrm{g}$ random hexamer; the reaction buffer as supplied by the manufacturer: $500 \mu \mathrm{M}$ dATP, dCTP, and dGTP; 300 $\mu \mathrm{M}$ dTTP; and $200 \mu \mathrm{M}$ amino-allyl dUTP. cDNA was purified using microcon-30 columns.

The cDNA containing amino-allyl dUMP residues was coupled to either Cy3 or Cy5 mono-reactive dyes (Amersham Pharmacia Biotech) in 0.05 M sodium bicarbonate at $\mathrm{pH} 9.0$, for $1 \mathrm{~h}$, before the reaction was quenched with $1.2 \mu \mathrm{M}$ hydroxylamine. Labeled cDNA from a sample and a reference was then mixed, and unincorporated dye was removed using a QIA-quick spin column (QIAGEN).

Chromosomal DNA for microarray experiments and for Southern blots was isolated using the DNeasy kit (QIAGEN) and digested with either AluI (microarrays) or PvuI (Southern blots). For microarrays, $2 \mu \mathrm{g}$ of DNA was mixed with $1 \mu \mathrm{g}$ random hexamer; $120 \mu \mathrm{M}$ dATP, dCTP, and dGTP; $60 \mu \mathrm{M}$ dTTP; $60 \mu \mathrm{M}$ Cy3- or Cy-5 labeled dUTP (Amersham Pharmacia); DNA polymerase Klenow fragment $(\mathrm{NEB})$; and the reaction buffer supplied by the manufacturer. After an overnight incubation at $37^{\circ} \mathrm{C}$, the labeled DNA was purified using a microcon30 column.

For EcoRI experiments, the reference DNA was from cells harvested prior to the temperature shift. For SwaI experiments, the reference RNA or DNA was from cells harvested before the addition of IPTG. For microarrays, the reference was labeled with Cy3 and the sample was labeled with Cy5. The mixture of Cy3- and Cy5-labeled DNA was hybridized to a microarray containing PCR products of at least $96 \%$ of the ORFs in the E. coli genome (Khodursky et al. 2003). Hybridization mixtures contained $4 \times$ SSC, $1 \%$ SDS, and $10 \mu \mathrm{g} / \mathrm{mL}$ rRNA. The hybridization occurred under a cover slip in a chamber humidified with one drop of $3 \times$ SSC for from $5 \mathrm{~h}$ to overnight at $63^{\circ} \mathrm{C}-65^{\circ} \mathrm{C}$. The microarrays were washed successively with $0.6 \times$ SSC plus $0.03 \%$ SDS, and $0.06 \times$ SSC. They were then spun dry in a swinging bucket centrifuge and scanned with a Genepix 4000B scanner (Axon) using the Genepix software (version 3.0).

\section{RNase protection assays}

To analyze quantitatively the expression of specific genes, the RPA III kit (Ambion) was used. The probes were $540 \mathrm{nt}$ of sequence antisense to the $\operatorname{dap} A$ transcript, $402 \mathrm{nt}$ sequence antisense to the xseA transcript, or $197 \mathrm{nt}$ sequence antisense to the $m r e B$ transcript. Each probe, in addition, contained $248 \mathrm{nt}$ from pBluescript that was subsequently degraded by the RNase. RPA products were run on a $5 \%$ denaturing polyacrylamide gel and quantified using a PhosphorImager.

\section{Electron microscopy and analysis of loop length}

E. coli chromosomes were purified as described (Worcel and Burgi 1972) with the following modifications. E. coli C600thy was grown to $\log$ phase at $30^{\circ} \mathrm{C}$ in minimal A medium plus $0.2 \%$ glucose, $40 \mu \mathrm{g} / \mathrm{mL}$ thr, $40 \mu \mathrm{g} / \mathrm{mL}$ leu, $80 \mu \mathrm{g} / \mathrm{mL}$ thy, and 1 $\mu \mathrm{g} / \mathrm{mL}$ thiamine. Minimal glucose medium was found to give cleaner preparations for microscopy than $\mathrm{LB}$, which was used elsewhere in this study. Cells $\left(10^{9}\right)$ were harvested for gentle lysis at room temperature in the presence of $80 \mathrm{U}$ RNasin (Pro- mega). Four-hundred microliters of lysate was layered directly on top of a 4-mL $10 \%-30 \%$ sucrose gradient containing $0.01 \mathrm{M}$ TrisHCl at $\mathrm{pH} 8.0,1 \mathrm{M} \mathrm{NaCl}, 1 \mathrm{mM}$ EDTA at $\mathrm{pH} 8$, and $1 \mathrm{mM}$ $\beta$-mercaptoethanol. Gradients were spun in an SW50.1 rotor of a Beckman ultracentrifuge for $25 \mathrm{~min}$ at 17,000 rpm. Eight microliters of the peak DNA fraction was placed directly on glowdischarged carbon-coated grids (Ted Pella) for $2 \mathrm{~min}$; the grids were then washed sequentially with $0.1 \mathrm{M}$ and $0.01 \mathrm{M}$ ammonium acetate, stained with uranyl acetate, and rotary shadowed with tungsten. Images were photographed on a JEOL 1200-EX microscope at 10,000- to 12,000-fold magnification.

Quantification of loop length was carried out on scanned negatives using SigmaScan Pro 5 (SSPS) with the trace measurement mode. Loops were traced from the base of the loop, where the loop emerges from the central mass of DNA. Calculated loop lengths were normalized to the measured length of a $7-\mathrm{kb}$ plasmid spread in sucrose gradient conditions. Five representative chromosomes were analyzed yielding 169 loop lengths. Evaluations by independent investigators were not significantly different. In addition, one chromosome was analyzed independently two times to estimate error. The average loop size between the two measurements differed by $<5 \%$, indicating a high degree of reproducibility in our measurements.

\section{Monte Carlo simulations}

To correlate gene expression with the domain structure of the chromosome, we performed Monte Carlo simulations. We implemented four different models of domain structure (Fig. 1A) and characterized them according to the expected resulting expression patterns. Domain barriers were modeled as single points. Models I and II assumed a constant distance between barriers with constant and varying placement between cells, respectively. Models III and IV were implemented similarly but assumed random positions of domain barriers.

Restriction was assumed to be complete. There were three interacting factors that determined the expression of SSGs: the positions of SSGs, restriction sites, and domain barriers. Since the position of one barrier determines the placement of all other barriers in fixed-length models (I and II), one extra adjustable parameter was needed: the placement of the initial domain barrier. In these cases, we verified that the simulation results were robust with respect to the position of the selected barrier by repeating the simulations with barrier placement at different positions. For each model, the number of barriers was varied to find the best-fit simulation to the experimental data.

The relative location of SSGs and restriction sites was determined using the E. coli MG1655 sequence (Blattner et al. 1997). Upon cleavage, only SSGs sharing a domain with a cleaved restriction site changed gene expression. Changes in expression of SSGs were modeled as independent Bernoulli random variables. This process was repeated until convergence was achieved ( 10,000 chromosomes). Gene expression changes for each SSG were averaged over all chromosomes.

\section{Acknowledgments}

We thank Adam Breier for help with data analysis and figure preparation. We thank Virgil Rhodius, Joyce West, Carol Gross, and Joe Derisi for help in preparing microarrays. Johannes Muelmenstaedt helped with statistical analysis and modeling. We thank N. Patrick Higgins for invaluable discussions and sharing unpublished results. Work from the authors' laboratory was supported by grants from the National Institutes of Health. C.D.H. is a Howard Hughes Medical Institute Pre-Doctoral fel- 
low. J.A. was supported by grant DMS 9971169 from the National Science Foundation.

The publication costs of this article were defrayed in part by payment of page charges. This article must therefore be hereby marked "advertisement" in accordance with 18 USC section 1734 solely to indicate this fact.

\section{References}

Aussel, L., Barre, F.X., Aroyo, M., Stasiak, A., Stasiak, A.Z., and Sherratt, D. 2002. FtsK Is a DNA motor protein that activates chromosome dimer resolution by switching the catalytic state of the XerC and XerD recombinases. Cell 108: 195-205.

Bernstein, J.A., Khodursky, A.B., Lin, P.H., Lin-Chao, S., and Cohen, S.N. 2002. Global analysis of mRNA decay and abundance in Escherichia coli at single-gene resolution using two-color fluorescent DNA microarrays. Proc. Natl. Acad. Sci. 99: 9697-9702.

Blattner, F.R., Plunkett III, G., Bloch, C.A., Perna, N.T., Burland, V., Riley, M., Collado-Vides, J., Glasner, J.D., Rode, C.K., Mayhew, G.F., et al. 1997. The complete genome sequence of Escherichia coli K-12. Science 277: 1453-1474.

Bliska, J.B. and Cozzarelli, N.R. 1987. Use of site-specific recombination as a probe of DNA structure and metabolism in vivo. J. Mol. Biol. 194: 205-218.

Case, R., Chang, Y., Smith, S., Gore, J., Cozzarelli, N., and Bustamante, C. 2004. The bacterial condensin MukBEF compacts DNA into a repetitive, stable structure. Science (in press).

Champoux, J.J. 2001. DNA topoisomerases: Structure, function, and mechanism. Annu. Rev. BioChem. 70: 369-413.

Clark, R.J. and Felsenfeld, G. 1971. Structure of chromatin. Nat. New Biol. 229: 101-106.

Delius, H. and Worcel, A. 1974. Electron microscopic studies on the folded chromosome of Escherichia coli. Cold Spring Harb. Symp. Quant. Biol. 38: 53-58.

Deng, S., Stein, R.A., and Higgins, N.P. 2004. Transcriptioninduced barriers to supercoil diffusion in the Salmonella typhimurium chromosome. Proc. Natl. Acad. Sci. 101: 3398 3403.

Drlica, K. 1987. The nucleoid. In Escherichia coli and Salmonella Typhimurium cellular and molecular biology. (ed. F.C. Neidhardt), pp. 91-103. American Society for Microbiology, Washington, D.C.

Espeli, O., Levine, C., Hassing, H., and Marians, K.J. 2003. Temporal regulation of topoisomerase IV activity in E. coli. Mol. Cell 11: 189-201.

Funnell, B.E., Baker, T.A., and Kornberg, A. 1986. Complete enzymatic replication of plasmids containing the origin of the Escherichia coli chromosome. J. Biol. Chem. 261: 56165624.

Gellert, M., O’Dea, M.H., Itoh, T., and Tomizawa, J. 1976. Novobiocin and coumermycin inhibit DNA supercoiling catalyzed by DNA gyrase. Proc. Nat1. Acad. Sci. 73: 4474-4478.

Gellert, M., Mizuuchi, K., O'Dea, M.H., Itoh, T., and Tomizawa, J.I. 1977. Nalidixic acid resistance: A second genetic character involved in DNA gyrase activity. Proc. Natl. Acad. Sci. 74: 4772-4776.

Heitman, J., Zinder, N.D., and Model, P. 1989. Repair of the Escherichia coli chromosome after in vivo scission by the EcoRI endonuclease. Proc. Natl. Acad. Sci. 86: 2281-2285.

Higgins, N.P., Yang, X., Fu, Q., and Roth, J.R. 1996. Surveying a supercoil domain by using the $\gamma \delta$ resolution system in Salmonella typhimurium. J. Bacteriol. 178: 2825-2835.
Hiraga, S., Niki, H., Ogura, T., Ichinose, C., Mori, H., Ezaki, B., and Jaffe, A. 1989. Chromosome partitioning in Escherichia coli: Novel mutants producing anucleate cells. J. Bacteriol. 171: 1496-1505.

Holmes, V.F. and Cozzarelli, N.R. 2000. Closing the ring: Links between SMC proteins and chromosome partitioning, condensation, and supercoiling. Proc. Natl. Acad. Sci. 97: 13221324.

Kavenoff, R. and Bowen, B.C. 1976. Electron microscopy of membrane-free folded chromosomes from Escherichia coli. Chromosoma 59: 89-101.

Kavenoff, R. and Ryder, O.A. 1976. Electron microscopy of membrane-associated folded chromosomes of Escherichia coli. Chromosoma 55: 13-25.

Khodursky, A.B., Bernstein, J.A., Peter, B.J., Rhodius, V., Wendisch, V.F., and Zimmer, D.P. 2003. Escherichia coli spotted double-strand DNA microarrays: RNA extraction, labeling, hybridization, quality control, and data management. Methods Mol. Biol. 224: 61-78.

Kong, H., Lin, L.F., Porter, N., Stickel, S., Byrd, D., Posfai, J., and Roberts, R.J. 2000. Functional analysis of putative restriction-modification system genes in the Helicobacter pylori J99 genome. Nucleic Acids Res. 28: 3216-3223.

Lindow, J.C., Britton, R.A., and Grossman, A.D. 2002. Structural maintenance of chromosomes protein of Bacillus subtilis affects supercoiling in vivo. J. Bacteriol. 184: 53175322.

Liu, L.F. and Wang, J.C. 1987. Supercoiling of the DNA template during transcription. Proc. Natl. Acad. Sci. 84: 70247027.

Lynch, A.S. and Wang, J.C. 1993. Anchoring of DNA to the bacterial cytoplasmic membrane through cotranscriptional synthesis of polypeptides encoding membrane proteins or proteins for export: A mechanism of plasmid hypernegative supercoiling in mutants deficient in DNA topoisomerase I. J. Bacteriol. 175: 1645-1655.

Manna, D. and Higgins, N.P. 1999. Phage Mu transposition immunity reflects supercoil domain structure of the chromosome. Mol. Microbiol. 32: 595-606.

Niki, H., Jaffe, A., Imamura, R., Ogura, T., and Hiraga, S. 1991. The new gene mukB codes for a $177 \mathrm{kd}$ protein with coiledcoil domains involved in chromosome partitioning of E. coli. EMBO J. 10: 183-193.

Pettijohn, D.E. 1996. The Nucleoid. In Escherichia coli and Salmonella: Cellular and molecular biology (ed. F.C. Neidhardt), pp. 158-156. American Society for Microbiology, Washington, D.C.

Poirier, M.G. and Marko, J.F. 2002. Mitotic chromosomes are chromatin networks without a mechanically contiguous protein scaffold. Proc. Natl. Acad. Sci. 99: 15393-15397.

Pruss, G.J. and Drlica, K. 1989. DNA supercoiling and prokaryotic transcription. Cell 56: 521-523.

Sawitzke, J.A. and Austin, S. 2000. Suppression of chromosome segregation defects of Escherichia coli muk mutants by mutations in topoisomerase I. Proc. Nat1. Acad. Sci. 97: 16711676.

Scheirer, K.E. and Higgins, N.P. 2001. Transcription induces a supercoil domain barrier in bacteriophage Mu. Biochimie 83: 155-159.

Schvartzman, J.B. and Stasiak, A. 2004. A topological view of the replicon. EMBO Rep. 5: 256-261.

Sherratt, D.J. 2003. Bacterial chromosome dynamics. Science 301: 780-785.

Sinden, R.R. and Pettijohn, D.E. 1981. Chromosomes in living Escherichia coli cells are segregated into domains of supercoiling. Proc. Natl. Acad. Sci. 78: 224-228. 
Sinden, R.R., Carlson, J.O., and Pettijohn, D.E. 1980. Torsional tension in the DNA double helix measured with trimethylpsoralen in living E. coli cells: Analogous measurements in insect and human cells. Cell 21: 773-783.

Trun, N.J. and Marko, J. 1998. The architecture of a functional chromosome. ASM News 64: 276-283.

Ullsperger, C., Vologodskii, A.V., and Cozzarelli, N.R. 1995. Unlinking of DNA by topoisomerases during DNA replication. In Nucleic Acids and molecular biology (ed. D.M. Lilley), pp. 115-142. Springer-Verlag, Berlin.

Vologodskii, A.V. and Cozzarelli, N.R. 1993. Monte Carlo analysis of the conformation of DNA catenanes. J. Mol. Biol. 232: $1130-1140$.

Worcel, A. and Burgi, E. 1972. On the structure of the folded chromosome of Escherichia coli. J. Mol. Biol. 71: 127-147.

Yu, D., Ellis, H.M., Lee, E.C., Jenkins, N.A., Copeland, N.G., and Court, D.L. 2000. An efficient recombination system for chromosome engineering in Escherichia coli. Proc. Natl. Acad. Sci. 97: 5978-5983.

Zechiedrich, E.L. and Osheroff, N. 1990. Eukaryotic topoisomerases recognize nucleic acid topology by preferentially interacting with DNA crossovers. EMBO J. 9: 4555-4562.

Zechiedrich, E.L., Khodursky, A.B., and Cozzarelli, N.R. 1997. Topoisomerase IV, not gyrase, decatenates products of sitespecific recombination in Escherichia coli. Genes \& Dev. 11: 2580-2592. 


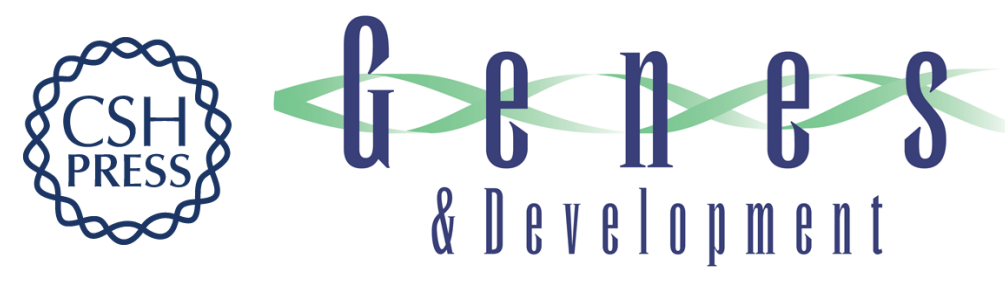

\section{Topological domain structure of the Escherichia coli chromosome}

Lisa Postow, Christine D. Hardy, Javier Arsuaga, et al.

Genes Dev. 2004, 18:

Access the most recent version at doi:10.1101/gad.1207504

References This article cites 39 articles, 20 of which can be accessed free at: http://genesdev.cshlp.org/content/18/14/1766.full.html\#ref-list-1

License

Email Alerting Receive free email alerts when new articles cite this article - sign up in the box at the top Service right corner of the article or click here.

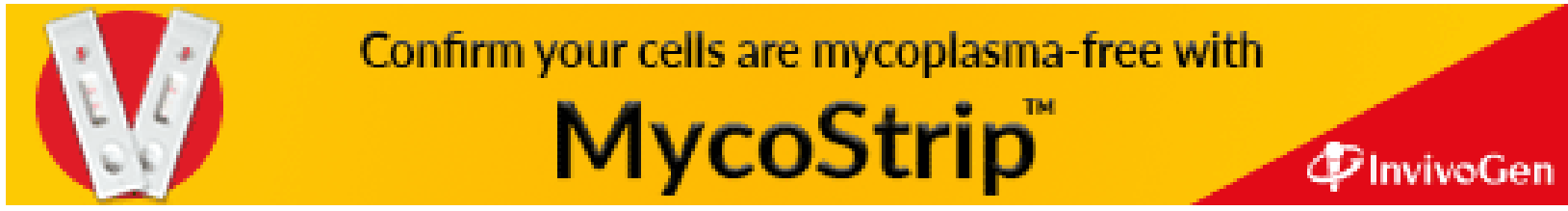

